\title{
Electrochemical Anodizing, Structural and Mechanical Characterization of Nanoporous Alumina Templates
}

\author{
A. P. Samantilleke ${ }^{1, a}$, J. O. Carneiro ${ }^{1, b}$, S. Azevedo ${ }^{1}$, T. Thuy $^{1}$ \\ and V. Teixeira ${ }^{1, c}$ \\ ${ }^{1}$ Department of Physics, University of Minho, Azurem Campus, 4810-058 Guimarães, Portugal \\ aanura@fisica.uminho.pt, 'carneiro@fisica.uminho.pt, 'vasco@fisica.uminho.pt
}

[Submitted: June 1, 2013; revised: July 25, 2013; accepted: July 25, 2013]

Keywords: AAO templates; Nanopores; two-step anodizing process; Nanoindentation;

\begin{abstract}
Highly ordered Anodic Aluminum Oxide (AAO) structures produced from aluminum by using an electrochemical anodizing method were developed towards its application for the next generation of micro/nano medical and energy devices. In addition of analyzing the anodizing current profile, the surface morphology was characterized by using Scanning Electron Microscopy (SEM), the crystalline structure by X-Ray Diffraction (XRD) and the mechanical properties by nanoindentation experiments. The anodizing time and applied potential determines the nanopores regularity and their size, although the effect of the potential is more pronounced than the effect of temperature in the transformation from crystalline alumina to amorphous alumina. Optimum pore growth was achieved with an applied potential of $17 \mathrm{~V}$ which led to a pore fraction - $P(f)$ - of about $17.5 \%$. The experimental Berkovich nanoindentation method was used to determine the AAO hardness as a function of the indenter depth, during the loading stage, using mechanical response and deformation behaviour of the nanopores structure. From the experimental data of the loaddisplacement curves, this method allows the calculation of the indenter contact depth at each reloading point, thus leading to the estimation of the material's hardness. The results reveal that the hardness depends on the processing conditions used for the production of the AAO samples that also strongly influences the organization and pore size uniformity.
\end{abstract}

\section{Introduction}

In recognition of the potential applications in nanoelectronics, energy and nanomedical fields, during the last decade, interest in one-dimensional nanomaterials such as nanotubes, nanorods and nanowires have significantly grown in both research and industrial sectors [1]. Templates consisting of nanopores with uniform diameter and length are regularly used to develop uniform nanostructured designs. The production of such templates holds the key to controlling the size and the shape of nanostructures [2]. Electrochemical production methods have been used to fill nanopores in the template with conducting, semiconducting or bio-molecular materials, in order to fabricate nanostructures and integrate them in circuit components [3]. Porous anodic aluminum oxides (AAO) have been attracting considerable attention in both the scientific and the commercial fields as an indispensable part of nanotechnology [4]. In 1970, O'Sullivan and Wood presented a model to describe self-regulating pore growth [5]. During the anodizing process within an acidic medium, the aluminum (Al) surface is oxidized where the oxide layer consists of a self-organized nanoporous structure. Interest in AAO nanostructures and their production using the two-step anodizing process has recently been revived, as described by Masuda and Fukuda [6]. A two-step anodizing process is believed to be essential in order to regulate the nanoporous structure, since irregular nanopores are formed on the Al substrate after the first anodizing step. Well-ordered nanopore arrays are obtained by etching out the oxide layer with irregularly arranged nanopores towards the film surface. Using this two-step method, and by applying a constant anodic voltage of $40 \mathrm{~V}$ in a $0.3 \mathrm{M}$ oxalic acid solution, Masuda and Fukuda obtained a well ordered nanopore array [6]. The plasticity model describes the formation of the anodic metal oxides, where it is believed to be a consequence of the flow of the film material in the barrier layer region beneath the porous 
layer. The material flows from the region of pore bases towards the cell wall regions due to growth stresses and field-assisted plasticity of the film material [7-9]. However, the formation mechanism of the pores is more complicated than previously thought [10]. Although the formation mechanism, pore ordering, inter-pore distance and pore size control have been extensively studied, there is much work still to be done in order to understand the electrochemistry and the anodizing process.

The applied potential in each anodizing process depends on the type of the electrolyte used. The effect of a variety of acids has been studied resulting in diverse degrees of success, which are determined by the pore ordering of the arrays obtained [11]. Sulphuric acid proves to be the most commonly used acid among the more effective electrolytes. When characterizing nanopore arrays, nanoindentation is a technique that provides invaluable information such as the hardness and the elastic modulus of the nanostructure under investigation [12]. Xia et al studied the effect of a heat treatment on AAO nanostructures and showed that increasing the temperature up to a maximum of $600{ }^{\circ} \mathrm{C}$ leaves the elastic modulus unaffected [13]. In this work, it is discussed the outcomes of the research studies into the mechanical properties of nanoporous AAO using nanoindentation. Furthermore, the mechanism of AAO template formation and structural properties were also determined and discussed.

\section{Experimental}

The nanopores AAO structures were electrochemically produced from $\mathrm{Al}$ sheet substrates by using a two-step anodizing process. The electrochemical setup comprised a $0.3 \mathrm{M} \mathrm{H}_{2} \mathrm{SO}_{4}$ acid, an anode (a pre-treated $\mathrm{Al}$ sheet) and a cathode (a Pb plate). The complete process for the synthesis of porous alumina is described below. Prior to anodizing, a chemical pre-treatment is undertaken in order to create micro-scale roughness on the Al substrate surface, as follows. After standard substrate cleaning, the Al foils (99\% purity) are subjected to a pre-treatment by ultra-sonication in ethanol/benzine for 10 to 15 minutes at room temperature. The $\mathrm{Al}$ foils are then dried and used as anodes in the electrochemical cell.

The first anodizing was carried out until the residual Al film thickness approaches the desired level, over a 60 minute period, then the oxides are removed by immersing the sample in a phosphoric acid $\left(6 \mathrm{wt} \% \mathrm{H}_{3} \mathrm{PO}_{4}\right)$ and chromic oxide $\left(1.8 \mathrm{wt} \% \mathrm{Cr}_{2} \mathrm{O}_{3}\right)$ mixture for a further 60 minutes at $60{ }^{\circ} \mathrm{C}$. In the second step, the remaining $\mathrm{Al}$ is anodized using the same conditions as the first anodizing but over a longer period of time (120 minutes) in order to achieve the desired thickness of the AAO layer. The final part of the experimental sequence involves etching in a solution of $\mathrm{H}_{3} \mathrm{PO}_{4}(5 \mathrm{wt} \%)$ at room temperature during 60 minutes (Table 1). For each anodizing step, the samples were immersed in the electrochemical deposition cell only when the temperature reaches $5( \pm 1){ }^{\circ} \mathrm{C}$, while rinsing with de-ionized water concludes each step. In this study, the current density was monitored during the anodizing procedure using a remotely controlled ammeter/voltage source. The conditions in which the samples were produced are given in Table 1.

Table 1 - Experimental conditions.

\begin{tabular}{|c|c|c|c|c|c|c|c|c|c|}
\hline \multirow{2}{*}{ SAMPLE } & \multicolumn{2}{|c|}{ Pre-treatment } & \multicolumn{2}{|c|}{$\begin{array}{c}\text { 1st anodizing } \\
{\left[\mathrm{T}=5 \pm 1^{\circ} \mathrm{C} \text {, Time }=60 \mathrm{~min} .\right]}\end{array}$} & \multirow{2}{*}{$\begin{array}{c}\begin{array}{c}\text { Etching of } \mathrm{Al}_{2} \mathrm{O}_{3} \\
{\left[\mathrm{~T}=60^{\circ} \mathrm{C}, \text { Time }=60\right.} \\
\text { min. }]\end{array} \\
\text { Chemical }\end{array}$} & \multicolumn{2}{|c|}{$\begin{array}{c}\text { 2nd anodizing } \\
{\left[\begin{array}{c}\mathrm{T}=5 \pm 1^{\circ} \mathrm{C}, \text { Time }=120 \\
\text { min. }]\end{array}\right.}\end{array}$} & \multicolumn{2}{|c|}{$\begin{array}{c}\text { Etching of } \mathrm{Al}_{2} \mathrm{O}_{3} \\
{[\mathrm{~T}=\mathrm{RT}]}\end{array}$} \\
\hline & Chemical & $\begin{array}{l}\text { Time } \\
\text { [min] }\end{array}$ & $\begin{array}{c}\mathrm{H}_{2} \mathrm{SO}_{4} \\
\text { Concentration } \\
{[\mathrm{M}]} \\
\end{array}$ & $\begin{array}{l}\text { Voltage } \\
{[\text { V] }}\end{array}$ & & $\begin{array}{c}\mathrm{H}_{2} \mathrm{SO}_{4} \\
\text { Concentration } \\
{[\mathrm{M}]} \\
\end{array}$ & $\begin{array}{c}\text { Voltage } \\
{[\text { V] }}\end{array}$ & Chemical & $\begin{array}{l}\text { Time } \\
{[\mathrm{min}]}\end{array}$ \\
\hline \multirow{2}{*}{ AAO (1-20) } & ethanol & 10 & 1 & \multirow{4}{*}{20} & \multirow{6}{*}{$\begin{array}{l}\mathrm{H}_{3} \mathrm{PO}_{4} 6 w t \% \\
\mathrm{Cr}_{2} \mathrm{O}_{3} 1.8 w t \%\end{array}$} & 1 & \multirow{4}{*}{20} & \multirow{6}{*}{$\begin{array}{l}\mathrm{H}_{3} \mathrm{PO}_{4} \\
5 \mathrm{wt} \%\end{array}$} & \multirow{6}{*}{60} \\
\hline & benzine & 15 & 1 & & & 1 & & & \\
\hline 1000250$)$ & ethanol & 10 & \multirow{4}{*}{0.3} & & & \multirow{4}{*}{0.3} & & & \\
\hline AAU (0.5-20) & benzine & 15 & & & & & & & \\
\hline \multirow{2}{*}{$\operatorname{AAO}(0.3-17)$} & ethanol & 10 & & \multirow{2}{*}{17} & & & \multirow{2}{*}{17} & & \\
\hline & benzine & 15 & & & & & & & \\
\hline
\end{tabular}

X-Ray diffraction (XRD) was performed using a Bruker AXS D8 Discover diffractometer, operated in $\theta-2 \theta$ geometry using $\mathrm{Cu}\left(\mathrm{K}_{\alpha}\right)$ radiation. XRD patterns were obtained with a step size of $0.02^{\circ}$ and an integration time of $1 \mathrm{~s}$. Scanning Electron Microscopy (SEM) imaging was performed with a FEI 
Nova 200FEG for all samples in order to evaluate the pore diameter and the inter-pore spacing. A Hysitron Nanoindentation system equipped with a Berkovich diamond indenter was used for the mechanical characterization.

\section{Results and Discussion}

Current density profile and pore formation. Fig. 1 shows a typical current density plot during the anodizing process, which consists of three noticeable current density regions related with the formation of porous alumina.

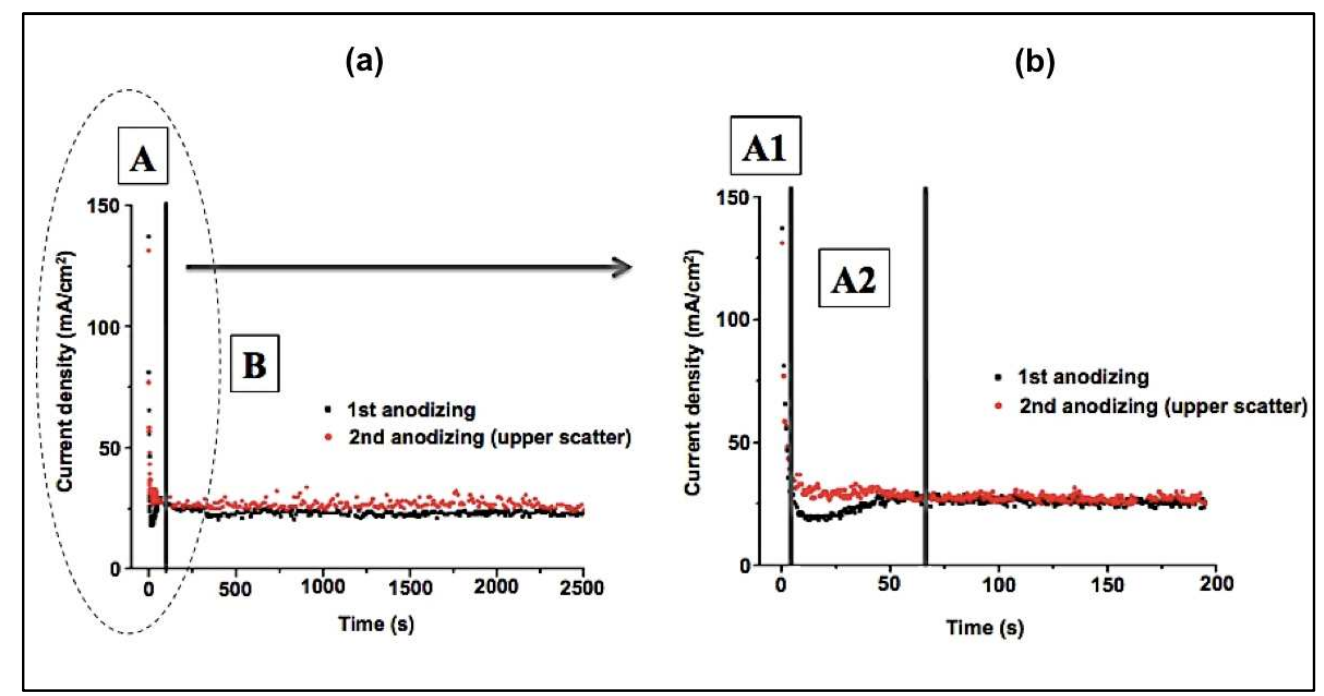

Fig. 1: (a) Current density $j(t)$ monitored during the two-step anodizing process in $0.3 \mathrm{M}$ sulfuric acid, $\left(U_{a \mathrm{p}}\right.$ $=20 \mathrm{~V}, \mathrm{~T}=5{ }^{\circ} \mathrm{C}$ ) presenting two clear regions; region A with varying current density and region $\mathrm{B}$ with a steady current density. (b) The region A where a sharp decrease in the current density is observed resulting from the growth of the oxide barrier layer (region A1). With this process, transport of $\mathrm{Al}^{3+}$ ions and their ejection to electrolyte results in a slow increase in the current density (region A2).

As has been previously reported, at the initial stage (region A), a barrier layer is first formed on the surface of the aluminum substrate when the potential is applied [14]. With the growth of the oxide barrier layer through the relatively fast reaction at the very early stages of the process (Eq. 1), the resistance of the electrode increases resulting in a sharp decrease in current density, as shown in the region $\mathrm{A} 1$ of the inset. With this process, the transport of $\mathrm{Al}^{3+}$ ions and their ejection to electrolyte results in a slow increase in the current density (region A2). However, steady current density is observed as shown in region $\mathrm{B}$, when equilibrium is achieved between the AAO formation and the $\mathrm{Al}^{3+}$ ion ejection. Singh et al attributed the pore initiation and self-organization of the porous structures at the initial stage to the considerable elastic stress in the oxide layer [15]. Although, the phenomenological description that the mechanical stress inflicted by the volume expansion on the structure due to the difference in ionic densities of $\mathrm{Al}^{3+}$ and $\mathrm{Al}$ in anodic alumina, a consequence of the difference in mass densities was possibly accountable for the self-organized formation of hexagonal pore arrays [16], the pore formation and the porous film growth is better described using the "Plastic ionic flow model". In this model, the pore formation in the anodized oxide is explained in terms of mass migration of metal ions within the oxide from underneath the pores to pore wall [79]. As described in the literature, barrier-type anodic alumina films grow by migration of $\mathrm{Al}^{3+}$ ions outward and $\mathrm{O}^{2-}$ ions inward [17]. As Thompson et al explained, about $40 \%$ of the film thickness forms at the AAO film/electrolyte interface as a consequence of the migration of $\mathrm{Al}^{3+}$ ions. The remainder of the film thickness is formed at the metal/film interface by migration of $\mathrm{O}^{2-}$ ions [18]. The formation of the porous film takes place when there is no film material being added at the film/electrolyte interface. While increased stresses from electrostriction assist stabilization of the pores, the absence of growth of new oxide at the film surface prevents pore filling.

Although, in general, the current densities of the first and the second anodizing step had similar overall behavior over time, some differences could be observed. The lowest current density was 
higher for the second anodizing step than for the first and the time taken to reach the constant current density during the second anodizing was shorter than that in the first step. This irregularity can be attributed to the fact that, in the first anodizing step, the porous structure is not yet formed and in the second anodizing the patterning of the pores has already taken place, after removing the aluminum oxide layer by etching in phosphoric acid solution $\left(\mathrm{H}_{3} \mathrm{PO}_{4}\right)$.

The formation of AAO itself occurs under an applied potential, following the surface oxidation, as more aluminum at the oxide-metal interface continues to oxidize (Eq. 1). Part of these $\mathrm{Al}^{3+}$ cations migrates across the oxide layer leading to the formation of a porous AAO structure. The migrated cations are released to the electrolyte where the liberated $\mathrm{Al}^{3+}$ are solvated and complexed. Thus, the main reaction that contribute to the porous AAO formations are:

$$
2 \mathrm{Al}+3 \mathrm{H}_{2} \mathrm{O} \rightarrow \mathrm{Al}_{2} \mathrm{O}_{3}+6 \mathrm{H}^{+}+6 e^{-} \text {(at the anode) }
$$

By another hand, the dissociation of water supplies anions to the oxide layer, but also plays an important role in governing the porosity of the porous AAO films [19].

Scanning electron microscopy and pore size analysis. The SEM micrographs presented in Fig. 2(a-c) show a well-defined, self-ordered, nanopored AAO structure obtained in this work.
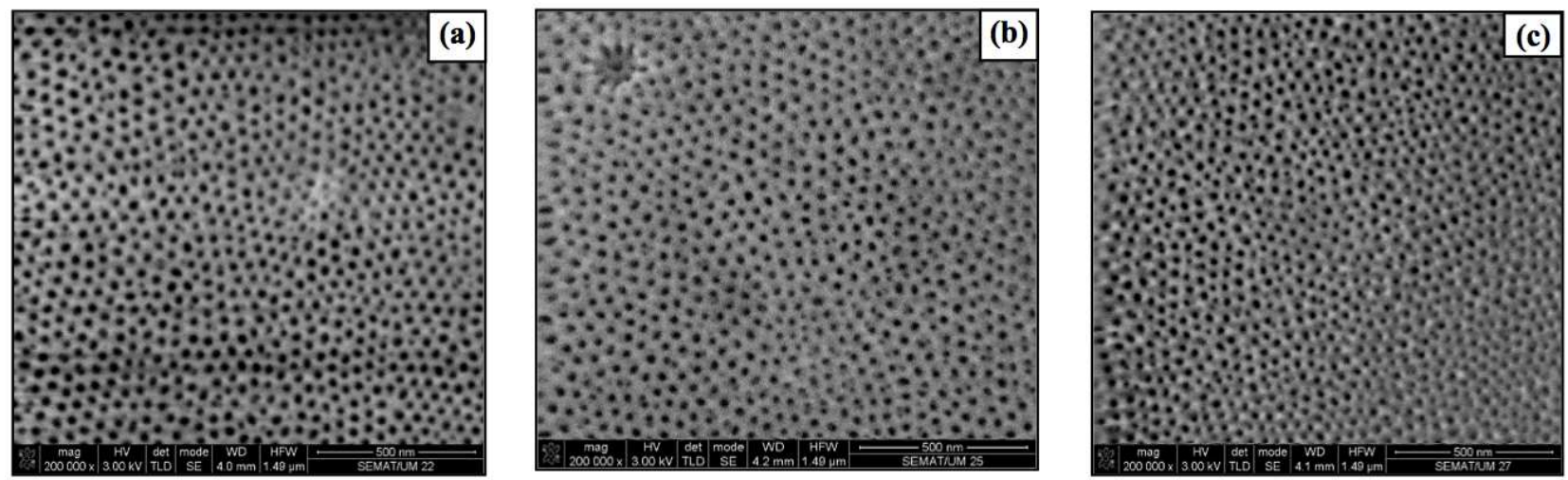

Fig. 2 - Surface SEM micrographs of AAO samples: (a) AAO (1-20), (b) AAO (0.3-20) and (c) AAO (0.317).

Samples AAO (1-20), AAO (0.3-20) and AAO (0.3-17) (see Table 1), anodized in different concentrations of electrolyte $(1 \mathrm{M}$ and $0.3 \mathrm{M})$, different applied potentials $(17 \mathrm{~V}$ and $20 \mathrm{~V})$ and at the same temperature $\left(5^{\circ} \mathrm{C}\right)$ have reasonably regular pores (Fig. 2). The samples presented pores diameters between 10 to $25 \mathrm{~nm}$ and an inter-pore distance of approximately $41 \mathrm{~nm}$. The nanosized pore structure is well developed presenting an uniform distribution of the nanopores. As described by Nielsch et al [20], the porosity or the pore fraction $P(f)$ of the AAO structure can be determined by using the Eq. 2 .

$$
P(f)=(2 \pi / \sqrt{3}) \cdot\left(r / D_{\text {int }}\right)^{2}
$$

where, $r$ and $D_{\text {int }}$ are the pore radius and the inter-pore distance respectively. Since a well defined AAO structure, $\left(r / D_{\text {int }}\right)$ is constant, and hence the optimum porosity calculated from Fig. 2, where $r$ $=9 \mathrm{~nm}$ and $D_{\mathrm{int}}=41 \mathrm{~nm}$, is $\sim 17.5 \%$. However, slight changes in the electrolyte concentration and temperature could shift the porosity due to the change in nature of acid attack on pore walls. Furthermore, the controlled adjustment of $r$ and $D_{\text {int }}$ by varying the applied potential has been observed in this work. When increasing voltage (or temperature), the pore diameter slightly increased being the variation insignificant. However, as Nielsch et al have shown, for alumina, the inter-pore distance, $D_{\text {int }}$, is linearly proportional to the applied potential $U$ (Eq. 3) with a proportionality constant $k$ of approximately $k \sim 2.5 \mathrm{~nm} / \mathrm{V}$. 


$$
D_{\text {int }}=k \boldsymbol{U}
$$

With an applied potential of $17 \mathrm{~V}$ for the AAO samples presented in Fig. $2, D_{\text {int }}=42.5 \mathrm{~nm}$ which correlates with the measured value of $41 \mathrm{~nm}$. In contrast, the average pore diameter remained constant for the two concentrations of sulphuric acid, suggesting the independent nature of the pore diameter from the acid concentration. As Masuda and Fukuda first reported self-organized pore growth leads to a densely packed hexagonal pore structure for certain sets of parameters as illustrated in Fig. 2.

AAO structure: X-ray diffraction (XRD) was employed to investigate the phase transformation of the AAO. In order to study the influence of temperature in the crystalline nature of the AAO structures, an additional sample (not presented in Table 1) was produced by using a electrolyte solution at $15{ }^{\circ} \mathrm{C}$. Fig. 3 shows the X-ray diffraction spectra of anodized aluminum with different temperature and voltage in the same electrolyte.

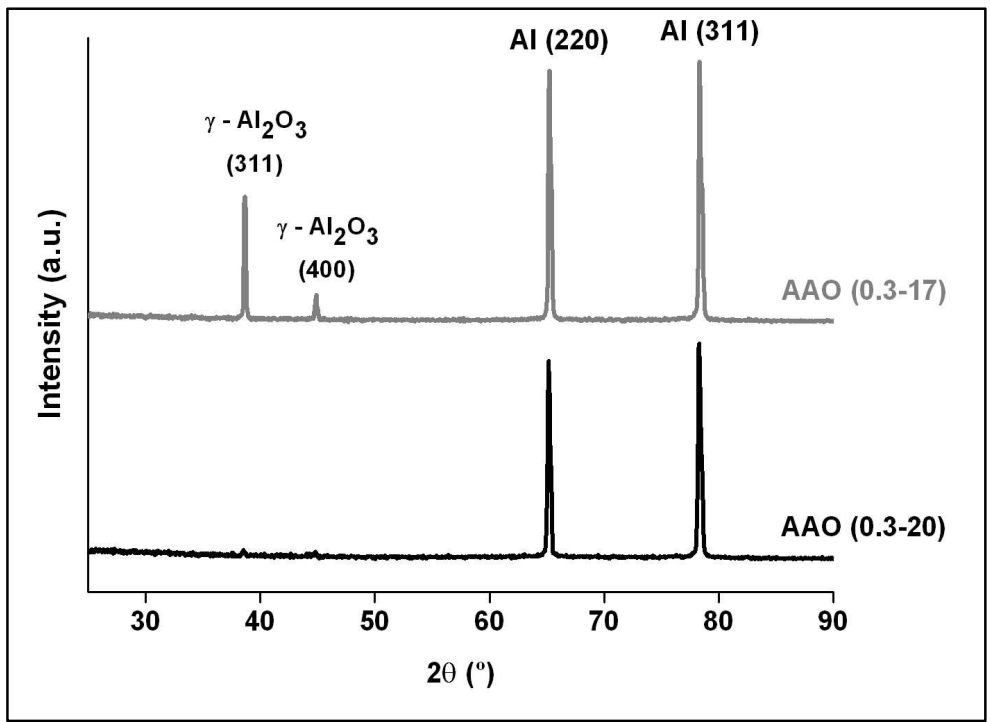

Fig. 3 - XRD spectra of AAO samples produced by using the electrolyte concentration and different applied voltage.

The (311) and (400) peaks of $\mathrm{Al}_{2} \mathrm{O}_{3}$ can be observed in the diffraction spectra of samples made under the same voltage $(17 \mathrm{~V})$ but different temperature conditions. However, the intensity of the two peaks is insignificant in the spectrum of the AAO sample made by applying a higher potential $(20 \mathrm{~V})$ in comparison with those made by applying a lower potential $(17 \mathrm{~V})$. The possible reason for this observation could be the formation of amorphous aluminum oxide under higher applied potentials. Higher intensities of the two peaks of $\mathrm{Al}_{2} \mathrm{O}_{3}$ can be seen from the sample made under low temperature in comparison with that made at higher temperature. However, it can be seen that the effect of the applied potential is stronger than the effect of temperature in the transformation of phase from crystalline alumina to amorphous alumina. The other diffraction peaks can be assigned to the aluminum substrate.

Nanoindentation. Nanoindentation has been widely used for measuring mechanical properties such as hardness and Young's modulus of samples. In a depth sensing indentation test, an indenter is pressed into the surface of a test solid and both the indenter load $\mathrm{P}$ and the indenter displacement $\mathrm{h}$ into the material are continuously recorded during the entire measurement. This provides a loaddisplacement curve $(P-h)$, which is a "fingerprint" of the mechanical properties of the tested material, from which the hardness and elastic modulus can be calculated. A Micro Materials Nanotest equipped with a Berkovich (triangular pyramid) diamond tip having a nominal edge radius of $\sim 100 \mathrm{~nm}$ was used in this study. 
A schematic representation of a typical indentation $(P-h)$ curve obtained with a Berkovich indenter is presented in Fig. 4(a).

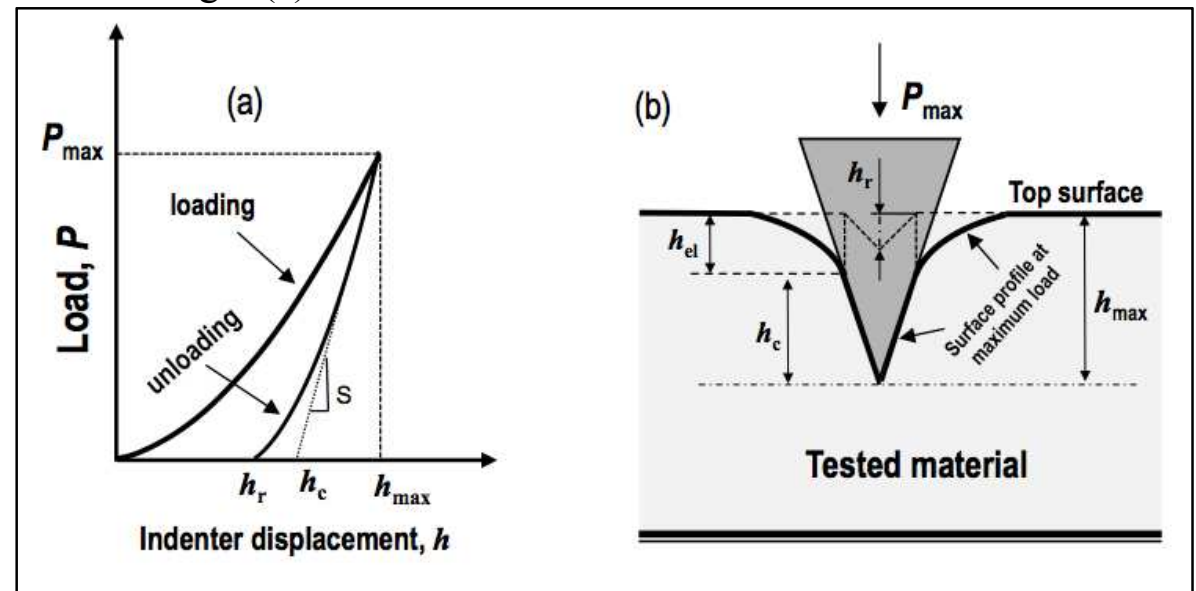

Fig. 4 - A schematic illustration of indentation load-displacement curve with several important parameters used in the Oliver and Pharr analysis: (a) loading/unloading cycle showing the main displacement parameters $h_{\mathrm{r}}, h_{\mathrm{c}}$ and $h_{\max }$ (b) sectional view of the physical parameters under maximum load: $h_{\max }$ (maximum indenter displacement), $h_{\mathrm{c}}$ (indenter contact depth) and $h_{\mathrm{r}}$ (residual depth after complete unloading).

There are three important quantities that can be measured from the $(P-h)$ curves: the maximum load, $P_{\max }$, the maximum displacement, $h_{\max }$, and the elastic unloading stiffness, $S=d P / d h$, defined as the slope of the upper portion of the unloading curve during the initial stages of unloading (also known as the contact stiffness). Another important parameter is the residual depth, hr, which is the permanent depression after the indenter is fully unloaded.

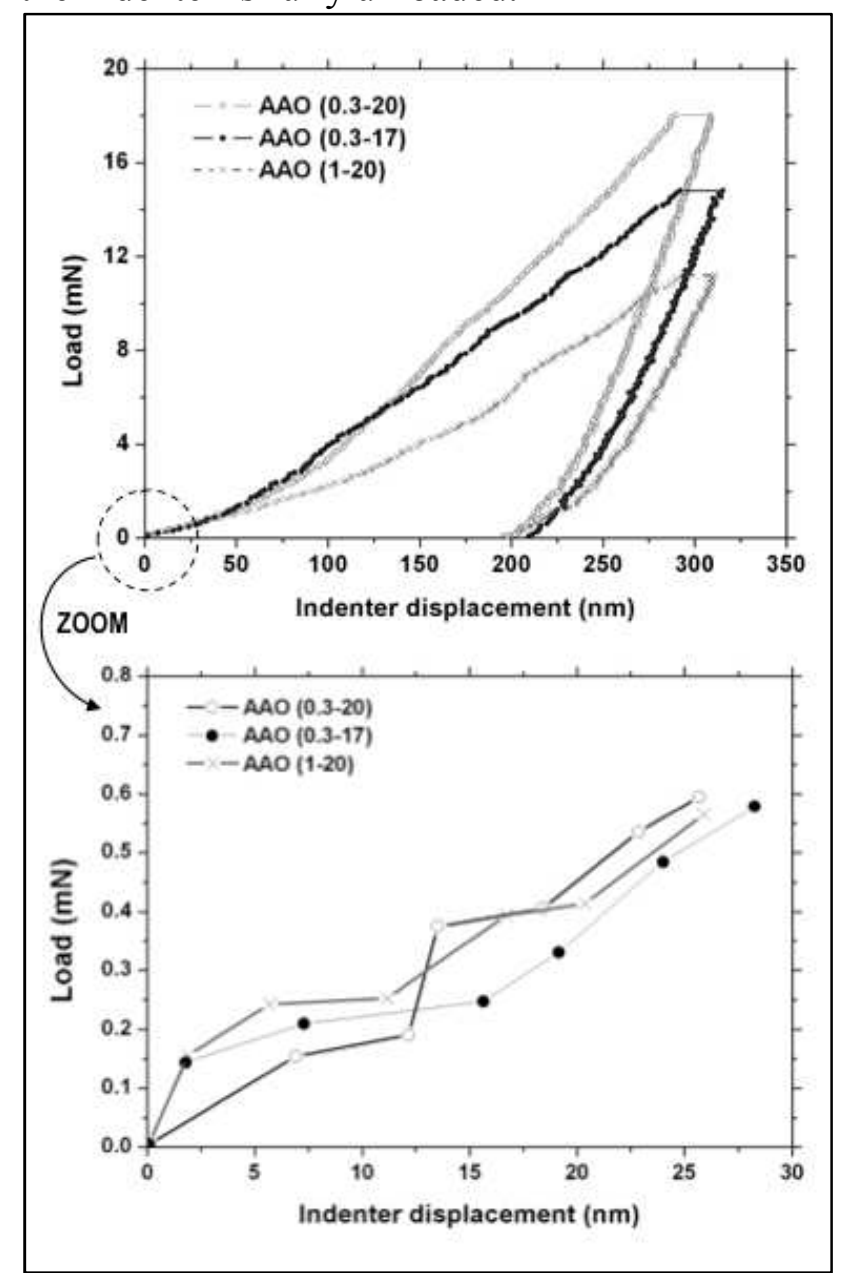

Fig. 5 - Load-displacement curves of the produced AAO samples: (a) one complete cycle of loading and unloading and (b) expanded view of the first loading steps. 
Fig. 4(b) shows the physical parameters of the indentation process in a cross sectional illustration in which it is assumed that the behavior of the Berkovich indenter, can be modeled by a conical indenter with a half- angle, $\theta=63.5^{\circ}$. The corresponding $(P-h)$ curves of the AAO samples, AAO (0.3-20), AAO (0.3-17) and AAO (1-20) with an indentation maximum displacement of $320 \mathrm{~nm}$ are shown in Fig. 5(a). The maximum displacement was limited to $320 \mathrm{~nm}$ in order to avoid the contribution from the non-oxidized Al metallic foils.

As the load increased, the indenter penetration displacement also increased (Fig. 5a). However, some small differences were noticeable. For example, for a given applied load (during the loading stage), the indenter displacement is not the same for all the analyzed AAO samples. In fact, it was observed that for a given applied load, the indenter did not penetrate the AAO (0.3-20) sample as much as it did in the other samples. This result suggests that the AAO (0.3-20) sample appears to be better able to withstand a state of deformation, which behavior is ascribed to the differences of the AAO pore sizes that are achieved by using different processing conditions. For a given applied load, the greater the pore diameter, the higher the material deformation is.

In addition, the results show (see Fig. 5b) that the pop-in behavior occurs at the beginning of the loading process. This behavior, which is different from the dislocation-slip phenomenon, as Fang et al described, is because the nanopore walls crash with one another [21]. In fact, a Berkovich diamond indenter with a radius of about $100 \mathrm{~nm}$ was used in this study. Although the indenter size is greater than the average pore or cell size, in the early stages of the applied load, the surface contact area between the indenter and the AAO material should be very small, which condition makes the average contact pressure higher than that of the rupture strength of the material, leading to the material collapse.

Furthermore, in order to characterize the mechanical properties of the AAO samples, it was determined the hardness and Young's modulus. The hardness, $H$, is the pressure exerted by the indenter at the maximum load $P_{\max }$ on the material, thus being determined from the Eq. 4 .

$$
\boldsymbol{H}=\frac{\boldsymbol{P}_{\max }}{\boldsymbol{A}}
$$

where $A$ is the projected (or cross sectional) area of the indenter at a distance $h_{c}$ back from its tip. The distance $h_{c}$ is the depth along which contact is made between the indenter and the sample. Under full load, the indenter is impressed to a total (maximum) depth $h_{\max }$, which can be taken from the load-displacement curve as indicated in the Fig. 4(a) and (b). After unloading is completed, there remains a residual depression of depth $h_{r}$, which is also given in the load-displacement curve.

Because the hardness is defined as being based on the projected contact area under load, it may not be as same as the conventional hardness (measured from the surface area of the residual hardness impression), if there is significant elastic recovery during unloading. The elastic modulus relates to contact area and the measured unloading stiffness through the Eq. 5 [22].

$$
S=\beta \frac{2}{\sqrt{\pi}} E_{r} \sqrt{A}
$$

where $E_{r}$, the 'reduced elastic modulus' (or the effective elastic modulus), is given by:

$$
\frac{1}{E_{r}}=\frac{1-v^{2}}{E}+\frac{1-v_{i}^{2}}{E_{i}}
$$

The reduced elastic modulus takes into account the fact that elastic displacements occur in both the sample and the indenter. The Young's modulus and the Poisson's ratio of the sample and the indenter $E, v$ and $E_{i}, v_{i}$ respectively. The properties of the diamond indenter used in this study are $E_{i}=1140 \mathrm{GPa}$ and $v_{i}=0.07$ [23] and the Poisson's ratio of the AAO samples is 0.22 [21]. 
The Eq. 5 is a general relation that applies to any axisymmetric indenter [24,25], where the dimensionless parameter, $\beta$, is used to account for deviations in stiffness caused by the lack of axial symmetry for pyramidal indenters. Indentation experiments are often conducted with nonaxisymmetric indenters. King [26] used finite element analysis to predict the $\beta$ values for different indenter geometries. He found that $\beta=1.034$ for a triangular punch, the value that has been widely adopted for instrumented indentation testing with a Berkovich indenter.

Although the Eq. 5 and Eq. 6 have been used for isotropic materials and a partial effect from other directions exists in the transversely isotropic case, it is still possible to apply those equations to estimate the elastic constant in the indentation direction.

The analysis used to determine the hardness, $H$, and elastic modulus, $E$, is an extension of the method proposed by Doerner and Nix [27] that accounts for the fact that unloading curves are distinctly curved in a way that cannot be explained by the flat punch approximation. Generally, in the flat punch approximation, the contact area remains constant as the indenter is removed, and the resulting unloading curve is linear. In contrast, experiments performed by Oliver and Pharr [28] has shown that unloading curves are distinctly curved and usually well fitted by a specific power law. The procedure used to measure $H$ and $E$, following the work performed by Oliver and Pharr, is based on the unloading process shown schematically in Fig. (4a), in which the elastic deflection, $h_{e l}$, of the sample surface at the maximum load is given by:

$$
\boldsymbol{h}_{e l}=\varepsilon \frac{\boldsymbol{P}_{\max }}{S}
$$

where $\varepsilon$ is a constant that depends on the geometry of the indenter and takes the numerical values of 1.00 for a flat punch, $\varepsilon=0.72$ for a conical punch, and $\varepsilon=0.75$ for a Berkovich indenter [22].

From the geometry of Fig. 4(b) that the depth along which, the contact is made between the indenter and the sample, $h_{c}=h_{\max }-h_{e l}$, is:

$$
h_{c}=h_{\max }-h_{e l}=h_{\max }-\varepsilon \frac{P_{\max }}{S}
$$

In order to determine the hardness of a material, Eq. 4 should be used. However, since the definition of hardness is based on the projected contact area under load, Oliver and Pharr [22] have defined an "area function", $F\left(h_{c}\right)$ that describes the projected (or cross sectional) area of the indenter at a distance, $h_{c}$, back from its tip. The area function is given by a multi-term polynomial fit of the form [22]:

$$
A=F\left(h_{c}\right)=\sum_{n=0}^{8} C_{n}\left(h_{c}\right)^{2-n}
$$

For a perfect pyramid or cone, the area function can be reasonably represented by the first term alone. By assigning the constant $C_{0}$ associated with the quadratic term to a value determined by the face angles of a Berkovich pyramid (with a half- angle of $65.3^{\circ}$ ), the resulting contact area is given by:

$$
A=\left(3 \sqrt{3} \tan ^{2} \theta\right) h_{c}^{2}=24.56 h_{c}^{2}
$$

Meanwhile, the monitoring of the average contact pressure is difficult due to the fact that the indenter displacement, rather than the contact depth, is measured during a nanoindentation test. The indenter displacement is the sum of the contact depth and the elastic deflection of the sample surface at the contact edge. It is therefore necessary to subtract the elastic deflection from each displacement measurement in order to calculate the average contact pressure. Oliver and Pharr 
technique, which is most often used to manipulate the nanoindentation test results, only provides the elastic deflection at the peak load.

Thus, to monitor the average contact pressure during reloading of the indenter, the procedure suggested by Novikov et al [29] and Carneiro et al [30] was used. The instantaneous value for the elastic deflection $h\left(e_{e l}\right)_{i}$ of the sample surface at contact perimeter was obtained as:

$$
\left(h_{e l}\right)_{i}=h_{e l}\left(P_{i} / P_{\max }\right)^{1 / 2}
$$

where $P_{i}$ is the instantaneous value of the applied load.

Using Eq. 11, the elastic deflection for all data points of the loading curve was calculated. The load dependence over the contact depth was found by subtracting the instantaneous elastic deflection from the instantaneous displacement data, according to the following equation.

$$
\left(\boldsymbol{h}_{c}\right)_{i}=\boldsymbol{h}_{i}-\boldsymbol{h}_{(e l) i}
$$

where $\left(h_{c}\right)_{i}$ is the instantaneous contact depth and $h_{i}$ the instantaneous displacement at each indentation reloading point.

The corresponding load-depth curve of the AAO (0.3-20) sample is shown in Fig. 6, which for comparison purposes, also includes the corresponding load-displacement curve presented previously in Fig. 5(a).

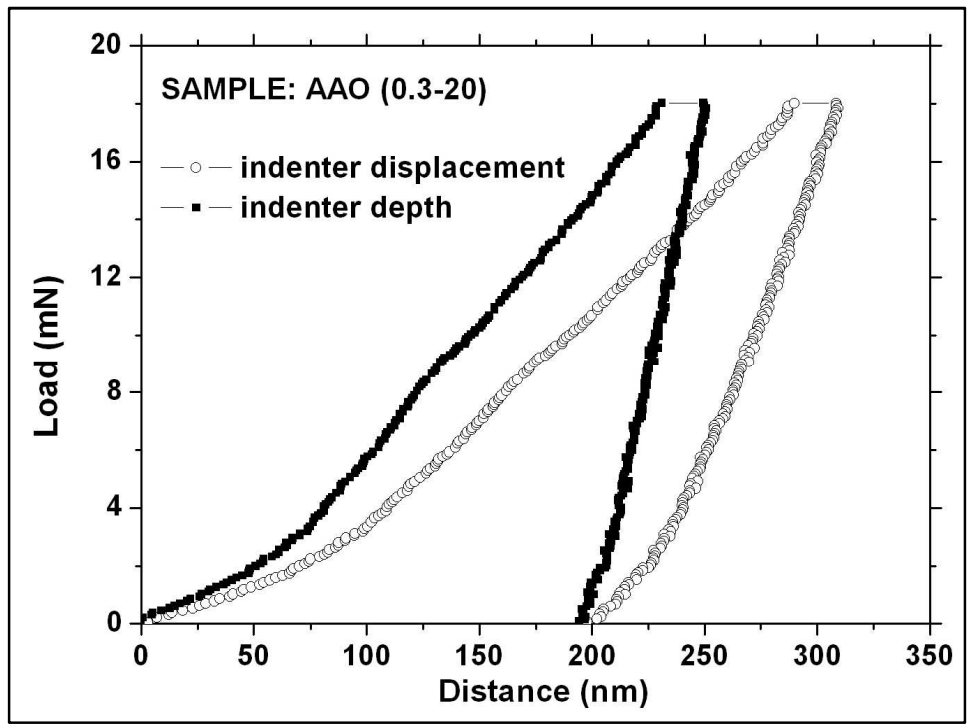

Fig. 6 - Comparison between the load-depth and load-displacement curves of AAO (0.3-20) samples.

It can be observed that the load-contact depth curve is shifted to the left side when compared with the load-displacement curve. This effect results from the subtraction of the elastic deflection of the test material from the displacement of the indenter. The greater the shift to the left, the more resilient is the material.

Meanwhile, the dependence of contact depth as well as the material surface elastic deflection over the applied load (under the loading process) is revealed in Fig. 7.

The surface elastic deflection of the tested sample increases slowly with the indenter displacement. However, for the same applied load, the magnitude of the contact depth is much more pronounced. In fact, at the peak load, the elastic deflection represents only $25 \%$ of contact depth. It can be noted that for an applied load smaller than $2.5 \mathrm{mN}$, the contact depth increases quickly as a result of the crash of nanopore walls. 


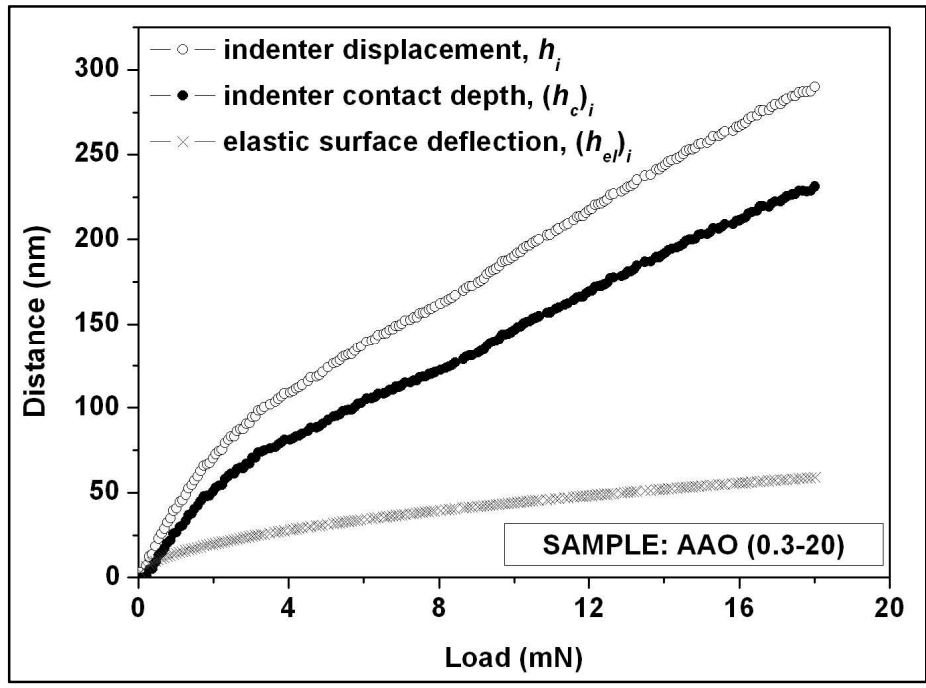

Fig. 7 - The dependence of contact depth, indenter displacement and the surface elastic deflection as a function of the applied load for AAO (0.3-20) sample. The surface elastic deflection of the tested samples increases slowly with the indenter displacement. For a small-applied load $(<2.5 \mathrm{mN})$, the contact depth increases rapidly that may result from the crash of nanopore walls.

Hardness as a continuous function of penetration depth can be calculated in two steps. At first, the elastic deflection of the sample surface at the peak load $h_{e l}$ was found using the Oliver and Pharr method. Then Eq. 12 was substituted in Eq. 4 in order to calculate the instantaneous hardness, $H_{i}$ (see Fig. 8).

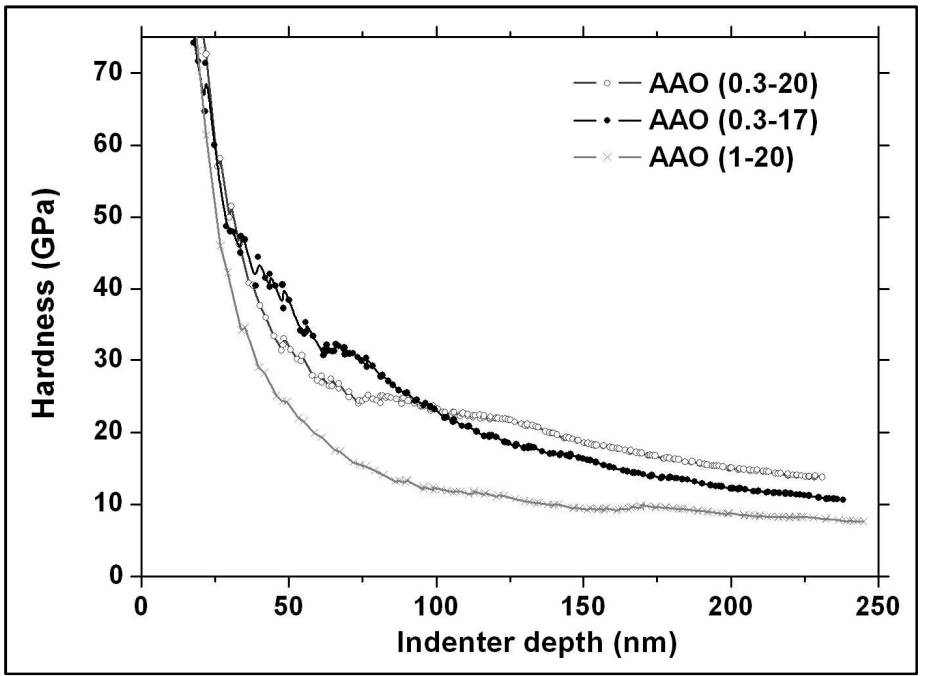

Fig. 8 - Hardness as a function of the indenter depth.

The hardness values are obtained from all loading curve data points and correspond to the ratio between the instantaneous applied loads and the instantaneous projected contact areas:

$$
(H)_{i}=\frac{P_{i}}{24.56}\left[h_{i}-\varepsilon\left(P_{\max } / S\right)\left(P_{i} / P_{\max }\right)^{1 / 2}\right]^{-2}
$$

Two regions can be highlighted on the hardness dependence over the indenter depth. In the first region, at a depth up to $75 \mathrm{~nm}$, a significant decrease in hardness is observed. The hardness decreased from $75 \mathrm{GPa}$ at a depth of $\sim 20 \mathrm{~nm}$ to $\sim 25 \mathrm{GPa}$ for samples AAO (0.3-20) and (0.3-17) and to $\sim 15 \mathrm{GPa}$ for sample AAO (1-20). However, from $\sim 75 \mathrm{~nm}$ to $245 \mathrm{~nm}$ the hardness decreases slowly with the indenter depth. Such behavior in hardness is due most probably to the fact that hardness measurements should be made (ideally) using sharp indenters. 
Since real pyramid indenters (such as Berkovich indenter) have a small rounded imperfection on its tip (inevitably occurs during its grinding process), for accurate measurements of hardness, the contact area between the indenter and the test material should be much larger when compared with the area of the defect at the indenter tip. This requirement is easy to attain for soft materials such as metals. However, for hard materials (oxides, carbides, nitrides etc.) at the beginning of an indentation experiment (very low applied loads), the area of the defect at the indenter tip can eventually be comparable to contact area. Under this condition, it is expected that small tip-rounded indenters can have some influence in the hardness calculations, since it can lead to the estimation of hardness with high magnitudes.

In fact, for the tested AAO samples, it was observed that for loads lower than approximately $2 \mathrm{mN}$, the hardness increases substantially. Meanwhile, as the contact depth increases, the contact area between the indenter and the AAO tested material should surpasses the size of defect at the indenter tip, and the hardness of the AAO material has decreased. This behavior may also result from the fragile and porous structure of the oxide material so that as the indenter penetrates the material, it is more and more cracked/broken to interior layers below the indenter tip.

On the other hand, for the region lying from about $75 \mathrm{~nm}$ to $245 \mathrm{~nm}$, the hardness remains almost constant in spite of the increase in indenter depth, therefore partly reflecting the mechanical properties of the crushed oxide material. The porous structure of the oxide material should lead to a deformation mechanism via crushed pores although the solid barrier layer may partially relieve the crushed pores underneath the indenter.

Moreover, for the region corresponding to the greater indenter penetration depths, the nearly constant hardness values suggest that the effect from the contribution of the substrate is not detected (since the penetration depth is much lower than that of the layer thickness). Fig. 8 also shows that the highest hardness value (calculated for maximum indenter penetration depth) was achieved for the AAO (0.3-20) sample, despite presenting a small difference when compared with the other samples. For the three tested samples, namely AAO (0.3-20), AAO (0.3-17) and AAO (1-20), the slight differences in hardness may be due to the different average pore diameters, $22 \mathrm{~nm}, 25 \mathrm{~nm}$ and $27 \mathrm{~nm}$ respectively (calculated from the imageJ software).

The hardness, $H$, and Young's modulus, $E$, of the AAO samples are presented in Table 2.

Table 2 - Average Hardness and Young's modulus calculated from indentation analysis.

\begin{tabular}{|c|c|c|}
\hline SAMPLES & Hardness [GPa] & Young's modulus [GPa] \\
\hline AAO (1-20) & 7.6 & 131.2 \\
\hline AAO (0.3-17) & 10.6 & 163.2 \\
\hline AAO (0.3-20) & 13.7 & 192.4 \\
\hline
\end{tabular}

The hardness of the sample AAO (0.3-20) is higher than that the sample AAO (1-20) and AAO $(0.3-17)$. This can be explained by the morphology of the surface of the samples, as previously mentioned.

The Young's moduli of the three nanoporous AAO samples, calculated for the highest indentation depth (of about $245 \mathrm{~nm}$ ), are $131.2 \mathrm{GPa}, 192.4 \mathrm{GPa}$ and $163.2 \mathrm{GPa}$, respectively. Moreover, the Young's moduli obtained here are slightly higher (of about 15\%) than those found elsewhere [31] because of the average pore diameter is lower than in the other study. Additionally, the values of the Young's modulus herein obtained may also be related with either absorbed moisture or residual water from the anodizing process, or the exposure to air.

\section{Conclusions}

Well-defined, self-ordered, porous alumina structures were obtained by a two-step electrochemical anodizing process of $\mathrm{Al}$ substrates. The applied potential affects the regularity and size of the nanopores and the effect of the potential appear to be stronger than the effect of 
temperature in the transformation from crystalline to amorphous alumina. While the inter-pore distance, is linearly proportional to the applied potential pore diameter remained independent of the electrolyte concentration. Optimum pore growth was achieved with a pore fraction of about $17.5 \%$. This experimental attained pore fraction probably needs further optimizing when improved pore ordering is required.

The mechanical properties of AAO structures were studied using nanoindentation measurements. It was observed that the AAO (0.3-20) sample, with the smallest pore diameter, presented the highest hardness value. The analysis of the hardness-contact depth data reveals that for the three tested samples, the indentation hardness decreased as the indenter penetration depth increased. Furthermore, for each of the tested samples, the corresponding hardness remains nearly constant in spite of the increase in indenter depth.

\section{Acknowledgements}

The authors would like to thank the FCT (Fundação para a Ciência e Tecnologia) for funding through Ciencia 2007 programme and the pluriannual contract with CFUM and the European Commission through FP7-PEOPLE-2010-IRSES-NanoCIS (269279) project.

\section{References}

[1] D.W. Thompson, P.G. Snyder, L. Castro, L. Yan, P. Kaipa, J.A. Woollam, Optical Characterization of Porous Alumina from Vacuum Ultraviolet to Mid-infrared, J. App. Phys. 97 (2005) 113511-113519.

[2] A.S. Aricò, P. Bruce, B. Scrosati, J-M. Tarascon, W.V. Schalkwijk, Nanostructured materials for advanced energy conversion and storage devices, Nature Mater. 4 (2005) 366 -377.

[3] J-M. Moon, A. J. Wei, Uniform Gold Nanorod Arrays from Polyethylenimine-coated Alumina Templates, Phys. Chem B. 109(49) (2005) 23336 - 23341.

[4] E. Moyen, L. Santinacci, L. Masson, W. Wulfhekel, M. Hanbücken, A Novel Self-Ordered Sub$10 \mathrm{~nm}$ Nanopore Template for Nanotechnology, Adv. Mater. 24 (37) (2012) 5094-5098.

[5] J.P. O'Sullivan, G.C. Wood, The Morphology and Mechanism of Formation of Porous Anodic Films on Aluminium, Proc. R. Soc A. 317(1970) 511-543.

[6] H. Masuda, K. Fukuda, Ordered metal nanohole arrays made by a two-step ... of honeycomb structures of anodic alumina, Science, 268 (1995) 1466-1468.

[7] G. Patermarakis, K. Moussoutzanis, Transformation of porous structure of anodic alumina films formed during galvanostatic anodising of aluminium, J. Electroanal. Chem. 659 (2011) 176-190.

[8] S.J. Garcia-Vergara, P. Skeldon, G.E. Thompson, H. Habazaki, A flow model of porous anodic film growth on aluminium, Electrochim. Acta. 52 (2006) 681-687.

[9] S.J. Garcia-Vergara, P. Skeldon, G.E. Thompson, H. Habazaki, Pore development during anodizing of Al-3.5 at.\%W alloy in phosphoric acid, Surf. Coat. Technol. 201 (2007) 9506-9511.

[10] Cheng Chuan.; A.H.W. Ngan, Modelling and simulation of self-ordering in anodic porous alumina, Electrochim. Acta. 56 (2011) 9998-10008.

[11] V. Sadasivan, C.P. Richter, L. Menon, P.F.Williams, Electrochemical self-assembly of porous alumina templates, AIChE Journal. 51(2) (2005) $649-655$.

[12] T.H. Fang, W.J. Chang, Nanomechanical Properties of Copper Thin Films on Different Substrates Using the Nanoindentation Technique, Microelectron. Eng. 65 (2003) 231-238.

[13] Z. Xia, L. Riester, W. Sheldon, W.A. Curtin, J. Liang, A. Yin, J.M. Xu, Mechanical Properties of Highly Ordered Nanoporous Anodic Alumina Membranes, Rev. Adv. Mater. Sci. 6 (2004)131139.

[14] X. Zhao, S-K. Seo, U-J Lee, K-H. Lee, Controlled Electrochemical Dissolution of Anodic Aluminum Oxide for Preparation of Open-Through Pore Structures, J. Electrochem. Soc. 154(10) (2007) C553-C557.

[15] G.K. Singh, A.A. Golovin, I.S. Aranson, Formation of Self-organized Nanoscale Porous Structures in Anodic Aluminum Oxide, Phys. Rev B. 73(20) (2006) 205422-1-205422-12. 
[16] O. Jessensky, F. Müller, U. Gosele, Self-organized formation of hexagonal pore arrays in anodic alumina, Appl. Phys. Lett. 72 (1998) $1173-1175$.

[17] F. Brown, W.D. Mackintosh, Use of Rutherford Backscattering to Study Behavior of IonImplanted Atoms During Anodic-Oxidation of Aluminum - Ar, Kr, $\mathrm{Xe}, \mathrm{K}, \mathrm{Rb}, \mathrm{Cs}, \mathrm{Cl}, \mathrm{Br}$, and I, J. Electrochem. Soc. 120 (1973) 1096.

[18] G.E. Thompson, Y. Xu, P. Skeldon, K. Shimizu, S.H. Han, G. C. Wood, Anodic oxidation of aluminium, Phil. Mag. B. 55 (1987) 651-667.

[19] Z.X. Su, G. Hahner, W.Z. Zhou, Investigation of the Pore Formation in Anodic Aluminium Oxide, J. Mater. Chem. 18(47) (2008) 5787-5795.

[20] K. Nielsch, J. Choi, K. Schwirn, R.B. Wehrspohn, U. Gösele, Self-Ordering Regimes of Porous Alumina: The 10\% Porosity Rule, Nano Lett. 2(7) (2002) 677-680.

[21] T-H. Fang, T.H.Wang, C-H. Liu, L-W. Ji, S-H. Kang, Physical Behavior of Nanoporous Anodic Alumina Using Nanoindentation and Microhardness Tests, Nanoscale Res. Lett. 2 (2007) 410-415.

[22] W.C. Oliver, G.M. Pharr, Measurement of Hardness and Elastic Modulus by Instrumented Indentation: Advances in Understanding and Refinements to Methodology, J. Mater. Res. 19 (1) (2004) 3-20.

[23] G. Simmons, H. Wang, Single Crystal Elastic Constant and Calculated Aggregate Properties: A Handbook second ed., The M.I.T. Press, Cambridge, Massachusetts, 1971.

[24] G.M. Pharr, W.C. Oliver, F.R. Brotzen, On the Generality of the Relationship among Contact Stiffness, Contact Area, and Elastic Modulus during Indentation J. Mater. Res. 7 (1992) 613-617.

[25] C-M. Cheng, Y-T. Cheng, On the Initial Unloading Slope in Indentation of Elastic-plastic Solids by an Indenter with an Axisymetrical Smoth Profile, Appl. Phys. Lett. 71 (1997) 2623-2625.

[26] R.B. King, Elastic Analysis of Some Punch Problems for a Layered Medium, Int. J. Solids Struct. 23 (1983) 1657-1664.

[27] M.F. Doerner, W.D Nix, A Method for Interpreting the Data from Depth-sensing Indentation Instruments, J. Mater. Res. 1 (1986) 601-609.

[28] W.C. Oliver, G.M. Pharr, An improved technique for determining hardness and elastic modulus using lod and displacement sensing indentation experiments, J. Mater. Res. 7 (1992), $1564-1583$.

[29] N.V. Novikov, S.N. Dub, Y.V. Milman, I.V. Gridneva, S.I Chugunova, Sverkhtverdye Materialy, 18 (1996) 37-46. (English translation, J. Superhard. Mater. Allerton Press Inc, New York 18(3): 32-40)

[30] J.O. Carneiro, V. Teixeira, A. Portinha, S.N. Dub, R. Shmegera, Hardness evaluation of nanolayered PVD coatings using nanoindentation, Rev. Adv. Mater. Sci. 7 (2004) 83-90.

[31] S. Ko, D. Lee, S. Jee, H. Park, K. Lee, W. Hwang, Mechanical properties and residual stress in porous anodic alumina structures, Thin Solid Films 515 (2006) 1932-1937. 
Journal of Nano Research Vol. 25

10.4028/www.scientific.net/JNanoR.25

Electrochemical Anodizing, Structural and Mechanical Characterization of Nanoporous Alumina Templates

10.4028/www.scientific.net/JNanoR.25.77 